PHYSICAL REVIEW D 93, 119905(E) (2016)

\title{
Erratum: On the description of nonunitary neutrino mixing [Phys. Rev. D 92, 053009 (2015)]
}

\section{F. J. Escrihuela, D. V. Forero, O. G. Miranda, M. Tórtola, and J. W. F. Valle}

(Received 17 May 2016; published 22 June 2016)

DOI: 10.1103/PhysRevD.93.119905

The constraint derived from the NOMAD experiment [1] was mistakenly reported in the published version of the paper. Our previous Eq. (38) should actually read

$$
\alpha_{11}^{2}\left|\alpha_{21}\right|^{2} \leq 0.0007 \quad \text { (90\% C.L.). }
$$

Combining this limit with those coming from universality at Eqs. (12) and (15), the following 90\% C.L. bounds (1 d.o.f.) are obtained:

$$
\alpha_{11}^{2} \geq 0.989, \quad \alpha_{22}^{2} \geq 0.999, \quad\left|\alpha_{21}\right|^{2} \leq 0.0007
$$
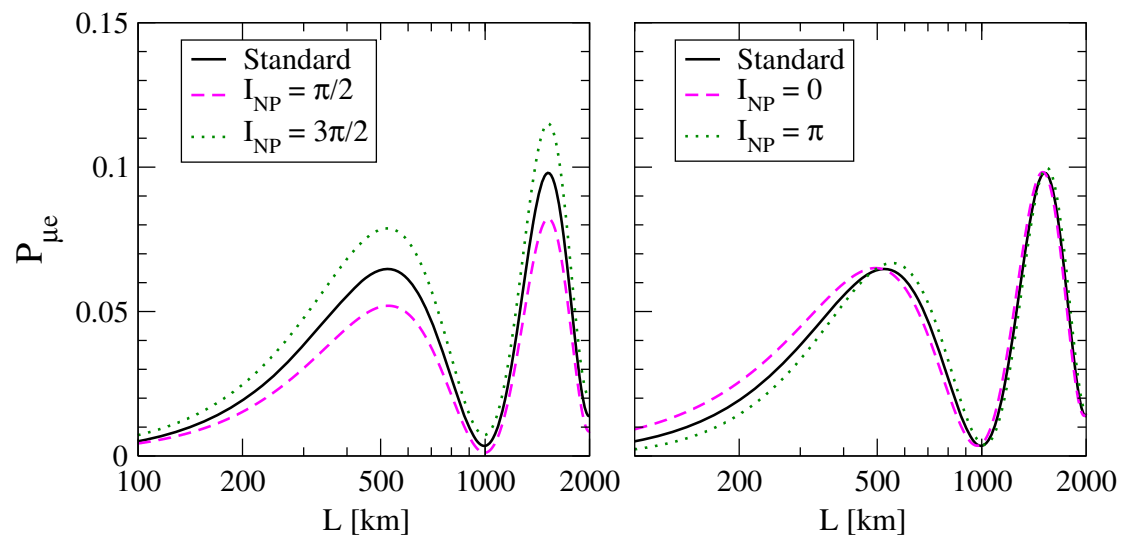

FIG. 1. Conversion probability for a fixed neutrino energy $E_{\nu}=1 \mathrm{GeV}$. The solid (black) curve shows the standard conversion probability, with $\delta=-I_{123}=3 \pi / 2$. The nonunitary case is illustrated for $\alpha_{11}=1, \alpha_{22}=0.9997$, and $\left|\alpha_{21}\right|=0.0264$. In the left panel, two values for the new $C P$ phase parameter $I_{N P}$ are considered: $\pi / 2$ (dashed/magenta line) and $3 \pi / 2$ (dotted/green line), while in the right panel we take $I_{N P}=0$ (dashed/magenta line) and $\pi$ (dotted/green line).
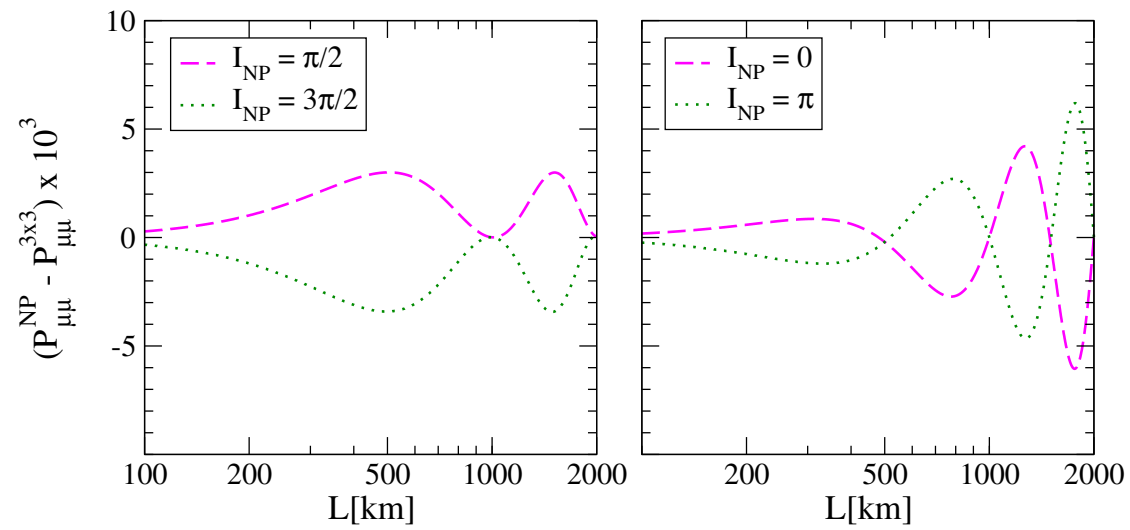

FIG. 2. Correction to the standard muon neutrino survival probability for different values of the new $C P$ phase parameter $I_{N P}$, with the remaining parameters fixed as in Fig. 1. 
Updated versions of Figs. 3 and 4 using the new reported bounds are shown here as Figs. 1 and 2. All the main conclusions of our article remain unchanged.

[1] P. Astier et al., Search for $\nu_{\mu} \rightarrow \nu_{e}$ oscillations in the NOMAD experiment, Phys. Lett. B 570, 19 (2003). 